\title{
MULTI-WAVELENGTH STUDY OF \\ ROSAT CLUSTERS OF GALAXIES
}

\author{
A. D. REID AND R. W. HUNSTEAD \\ School of Physics, University of Sydney \\ NSW 2006, Australia \\ AND \\ M. M. PIERRE \\ CEA/DSM/DAPNIA CE Saclay, France
}

We are engaged in a radio-IR-optical-X-ray study of two flux-limited samples of ROSAT clusters of galaxies south of declination $-20^{\circ}$ (Pierre et al., 1994a). One sample covers an area of $1750 \mathrm{deg}^{2}$ in Hydra (Pierre et al., 1994b) and includes some distant $(z \approx 0.3)$ and X-ray luminous $\left(L_{X} \approx 10^{45} \mathrm{erg} \mathrm{s}^{-1}\right)$ clusters. The other sample derives from a volumelimited subset (nominally $z \leq 0.1$ ) of southern X-ray clusters, which are the focus of an ESO Key Program (Guzzo et al., 1995).

An observing program with the Molonglo Observatory Synthesis Telescope (MOST) at $843 \mathrm{MHz}$ and the Australia Telescope Compact Array (ATCA) at 1.4 and $2.4 \mathrm{GHz}$ has been undertaken to complement existing optical spectroscopy and photometry (ESO and CFHT), pointed X-ray (ROSAT PSPC and HRI) images, and scheduled FIR (ISO) observations.

Our radio observations, together with data at other wavelengths, are helping to give a more comprehensive picture of the cluster environment. As an example, we have chosen a cluster in the Hydra region, ROSAT RXJ $1254.4-2901$, part of the A3528 complex at $z=0.0535$ and located in an important region of the sky, the Shapley 8 supercluster. In Figure 1 we show a preliminary montage of images for this cluster (Pierre et al., 1995, in prep.). There is a strong radio source (A: $0.59 \mathrm{Jy}$ total at $843 \mathrm{MHz}$ ) coincident with the $\mathrm{X}$-ray centroid, suggesting that non-thermal emission from an active nucleus may be contaminating the extended thermal $\mathrm{X}$ ray emission from the intracluster medium. MOST detects an additional source (B: $0.52 \mathrm{Jy}$ total) south of the X-ray centroid but still within the $\mathrm{X}$-ray envelope. 


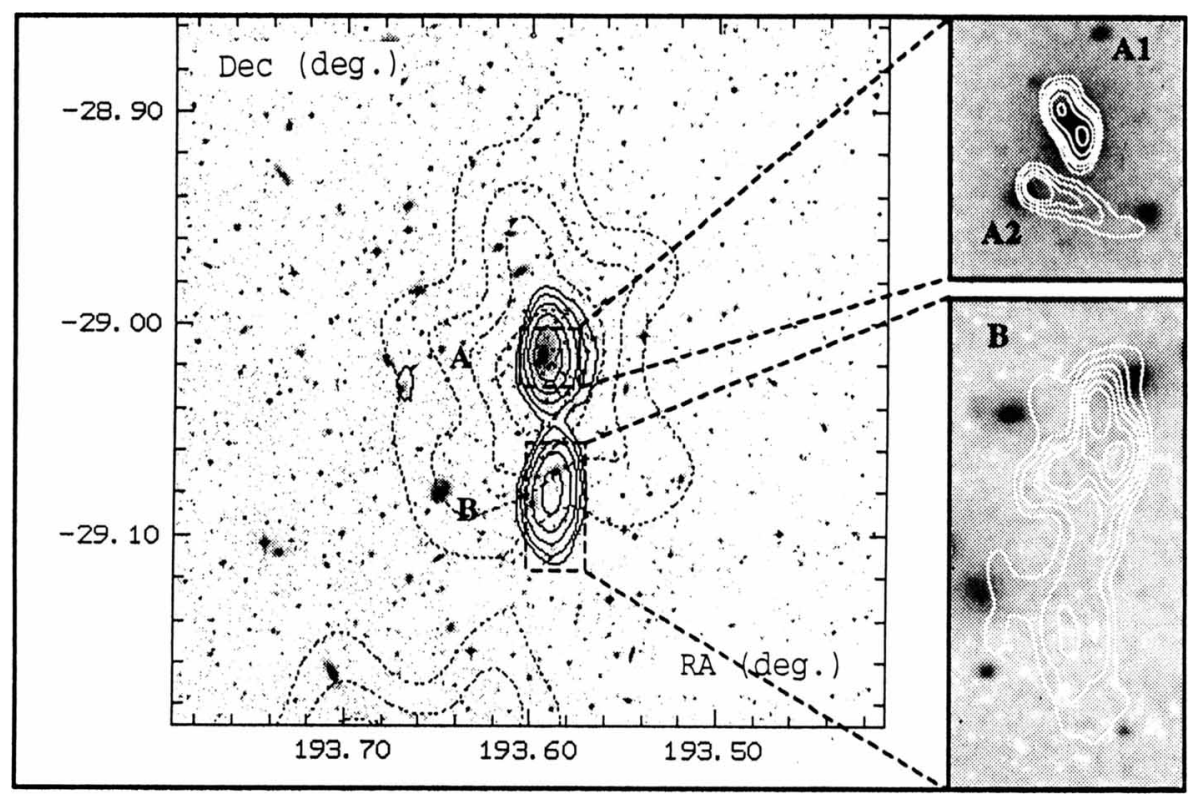

Figure 1. Multi-wavelength overlay in the region of A3528; coordinates are for equinox J2000. Left: X-ray contours of RXJ 12 54.4 -29 01 are shown dotted and MOST contours as continuous, against a background of the NASA/STScI digitized sky survey. Right: ATCA contour images of source $A$ at $2.4 \mathrm{GHz}$ and $\mathrm{B}$ at $1.4 \mathrm{GHz}$ are shown in white, superposed on smoothed greyscale images from the DSS.

The ATCA images reveal considerable structure in both these sources. At $2.4 \mathrm{GHz} \mathrm{A}$ is clearly resolved into two separate sources. The northern component $\mathrm{A} 1$ is a close double, possibly a wide-angled tail source, coincident with the $\mathrm{cD}$ galaxy. The southern component $\mathrm{A} 2$ has a head-tail morphology and is most likely identified with the bright galaxy lying close to the radio peak. These identifications are supported by lower-resolution 4.8 GHz VLA images (Gregorini et al., 1994). Source B, which is relatively diffuse, appears to have a narrow-angled tail structure associated with the bright galaxy just to the NW of the two emission peaks. The implied direction of motion is towards the $\mathrm{X}$-ray centre and source $\mathrm{A}$.

\section{References}

Gregorini, L., de Ruiter, H.R., Parma, P., Sadler, E.M., Vettolani, G. \& Ekers, R.D. (1994), $A \& A S, 106,1$.

Guzzo, L. and 26 others (1995) in Wide-Field Spectroscopy and the Distant Universe, eds. S. J. Maddox and A. Aragón-Salamanca (World Scientific: Singapore), p. 205.

Pierre, M., Hunstead, R., Reid, A. and 10 others (1994a), ESO Messenger, 78, 24.

Pierre, M., Böhringer, H., Ebeling, H., Voges, W., Schuecker, P., Cruddace, R. \& MacGillivray, H. (1994b), $A \& A, 290,725$. 